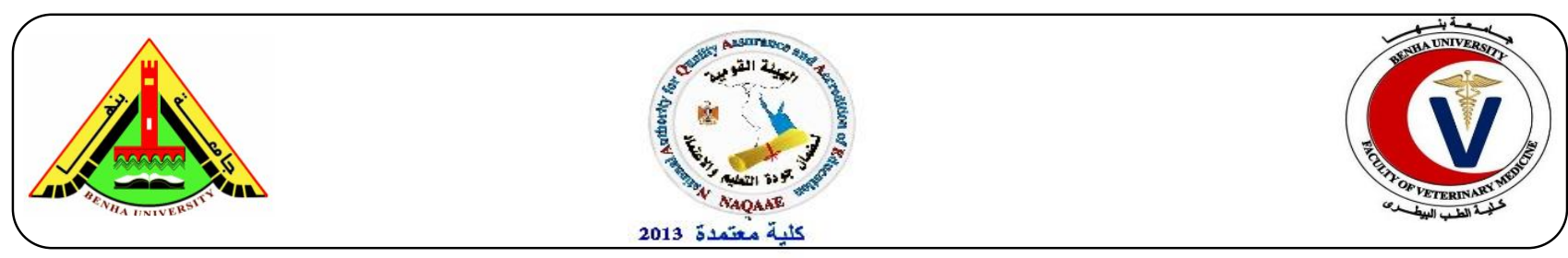

\title{
The Potential Protective Effect of Spirulina Platensis against Mycotoxin Induced Oxidative Stress and Liver Damage in Rats
}

\author{
Hussein S.A. ${ }^{*}$; Abd el-hamid O.M. ${ }^{1}$ El-tawil O.S. ${ }^{2}$,Laz E.S. $^{3}$ and Taha W.M. ${ }^{3}$ \\ ${ }^{1}$ Department of Biochemistry, Faculty of Vet. Med., Benha University, Egypt. \\ ${ }^{2}$ Department of Toxicology and Forensic Med., Faculty of Vet. Med., Cairo University, Egypt. \\ ${ }^{3}$ Department of Biochemistry and food deficiency, Animal Health Research Institute, Dokki, Giza,
} Egypt.

*Corresponding author: Samy Ali Hussein; email: samyaziza@yahoo.comsamy.aziza@Futm.bu.edu.eg

\section{A B S T R A C T}

The present study was conducted to evaluate the protective effect of Spirulina on mycotoxins induced liver damage and oxidative stress in rats. Thirty male albino rats were classified randomly into three equal groups. Group1(normal control): Rats fed with ordinary ration. Group2 (Mycotoxin): Rats fed with ordinary ration only for first 7 days and from day 8 rats fed mixed ration contain 50 gram of grinded plant (total aflatoxins $4.2 \mathrm{ppb}$ ) +50 gram of grinded fish (total aflatoxins $42.14 \mathrm{ppb})+50$ gram of ordinary diet (free of aflatoxins) for 4 weeks. Group3 (Mycotoxin+ Spirulina): Spirulina Platensis (500 mg/kg b.wt/day) was administered orally allover the experimental period (5 weeks). Also, rats were fed on ordinary diet only for first 7 days and from day 8 rats fed mixed ration as that mentioned above in group 2. At the end of the experiment liver tissue specimens were isolated and analyzed for the determination of reduced glutathione (GSH), superoxide dismutase (SOD), catalase (CAT), L-malondialdehyde (L-MDA) in addition to intrerleukin-1(IL-1) gene expression and DNA damage. The obtained results showed a significant up-regulation of IL-1 gene expression level and marked increase in SOD, CAT, L-MDA and DNA damage that was indicated by an increase in tail length and tail DNA \% in liver tissue of mycotoxin rats group. However, liver GSH concentration was markedly decreased as compared to control group. Spirulina protected mycotoxins induced liver damage in rats caused a significant improvement of all previous parameters and attenuates DNA changes. Conclusively, Spirulina Platensis treatment exerts a protective effect against DNA damage and oxidative stress in liver of mycotoxin intoxicated rats through free radical scavenging and antiinflammatory activities as well as regenerating endogenous antioxidants defense system mechanisms.

Key words: Mycotoxins, Liver damage, Spirulina platensis, Oxidative stress.

(http://www.bvmj.bu.edu.eg)

(BVMJ-35(2): 375-383, 2018)

\section{INTRODUCTION}

Mycotoxins are secondary metabolites produced by a wide range of fungi known to contaminate a variety of food and agricultural commodities worldwide. Their occurrence in food, beverages and feed has been recognized as potential threat to humans and animals, 
either by direct contamination of plant materials or products (Fink-Gremmels, 1999), or by "carry over" of mycotoxins and their metabolites into animal tissues, milk and eggs after intake of contaminated feed. Mycotoxins have carcinogenic, mutagenic, teratogenic, immunosuppressive, and tremorgenic effects (Ayçiçek et al., 2005). Over 400 mycotoxins are known and the foodborne toxins of most interest are aflatoxins B1 (AFB1), aflatoxins B2 (AFB2), aflatoxins G1 (AFG1), aflatoxins G2 (AFG2), trichothecenes, fumonisins(FB1 and FB2), ochratoxin A (OTA), zearalenone (ZEN) and Alternaria toxins, due to their frequent occurrence and their severe effects on animal and human health (Hussein and Brasel, 2001).

Spirulina (SP), is symbiotic, multicellular and filamentous cyanobacterium (blue-green alga), that has free-floating filamentous microalgae with spiral characteristics of its filaments and symbiotic bacteria that fix nitrogen from air. It is formally called Arthrospira, belonging to Phylum: Cyanobacteria, Class: Cyanobacteriaceae, Order: Nostocales, Family: Oscillatoriaceae, Genus: Spirulina with characteristic photosynthetic capability (Komárek et al., 2009). Among large number of Spirulina species, three species of Spirulina, including Spirulina platensis (Arthrospira platensis), Spirulina maxima (Arthrospira maxima) and Spirulina fusiformis (Arthrospira fusiformis) are most intensively investigated as those Spirulina species are edible with high nutritional as well as potential therapeutic values. One of these, Arthrospira platensis is the most common and widely available spirulina and most of the published research and public health decision refers to this specific species (Karkos et al., 2008). Spirulina contains proteins (up to $70 \%$ protein of dry weight), lipids (contain 5-8\% lipid), carbohydrates( up to $14 \%$ ), some vital minerals (like calcium, iron, zinc, magnesium, manganese, selenium), essential fatty acids, polysaccharides, glycolipids (contain 40\%), sulpholipids (2-5\%), vitamins including B-carotene, a precursor of vitamin A, substantial amounts of carotenes stimulate the immune system and reduce toxic effects of radiation and chemotherapy (Cohen, 1997).

Accordingly, the present study was carried out to investigate the harmful exposure effect of mycotoxins in rats and trials for ameliorating these mycotoxins effect using blue green algae (Spirulina).

\section{MATERIALS AND METHODS}

\subsection{Experimental animals:}

Thirty white male albino rats of 45 weeks old and average body weight 100$130 \mathrm{~g}$ were used in this study. Rats were obtained from Laboratory Animals Research Center, Animal health research animal institute, Dokki, Cairo. Rats were housed in separated polycarbonate cages (10 per cage) and they were kept on a well-balanced ration and fresh clean drinking water ad-libitum. Rats were kept at a constant environmental and nutritional condition throughout the whole period of experiment. All rats were left for 15 days for acclimatization before the beginning of the experiment.

\subsection{Natural medical plant:-}

\subsubsection{Spirulina platensis}

Spirulina microalgae blue green powder was obtained from Cairo National Research Center - Dokki-Egypt.

-Preparation and Dosage of Spirulina:

Spirulina was freshly prepared by dissolved in distilled water and administered orally using stomach tube in a daily dose of $500 \mathrm{mg} / \mathrm{kg}$ body weight (Colla et al., 2008).

\subsection{Experimental design:-}

After acclimatization to the laboratory 
conditions, the animals were randomly classified into three groups (10 rats each) placed in individual cages and classified as follow:

Group 1 (control group): Rats fed with ordinary diet (free of mycotoxin) only without any treatment during the entire experimental period of 5 weeks.

Group 2 (Mycotoxin group):Rats fed with ordinary diet only for first 7 days and from day 8 rats fed mixed ration contain 50 gram of grinded plant (total aflatoxins $4.2 \mathrm{ppb}$ ) +50 gram of grinded fish (total aflatoxins 42.14 $\mathrm{ppb}$ ) +50 gram of ordinary diet (free of aflatoxins) for 4 weeks.

Group 3 (mycotoxin + Spirulina group): Spirulina Platensis (500 mg/kg b.wt/day) was administered orally allover the experimental period (5 weeks). Also, rats fed with ordinary diet only for first 7 days and from day 8 rats fed mixed ration contain 50 gram of grinded plant (total aflatoxins $4.2 \mathrm{ppb}$ ) +50 gram of grinded fish (total aflatoxins $42.14 \mathrm{ppb}$ ) +50 gram of ordinary diet (free of aflatoxins) for 4 weeks.

\subsection{Sampling:}

Liver tissue specimens were collected from all animal groups (control and experimental groups) once after the end of 5 weeks.

\subsubsection{Tissue samples for biochemical analysis:-}

At the end of the experiment the rats were sacrificed under light ether anesthesia. Liver tissues were isolated immediately, weighed and then one of them was cleaned by rinsing with cold saline and stored at -20 ${ }^{\circ} \mathrm{C}$ for subsequent biochemical analyses. All liver samples were analyzed for the determination of reduced Glutathione (GSH), superoxide dismutase (SOD), catalase (CAT) and L-malondialdehyde (L-MDA).

2.4.1.1. Liver tissue preparation for biochemical analysis:-
Liver tissues were cut, weighed and minced into small pieces, homogenized with a glass homogenizer in 9 volume of ice-cold $0.05 \mathrm{mM}$ potassium phosphate buffer ( $\mathrm{pH} 7.4)$ to make $10 \%$ homogenates. The homogenates were centrifuged at 6000 r.p.m for 15 minutes at $4{ }^{\circ} \mathrm{C}$ then the resultant supernatant were used for the determination of the following parameters: L-MDA, SOD and CAT.

About $0.2 \mathrm{~g}$ of liver tissues were minced into small pieces homogenized with a glass homogenizer in $0.4 \mathrm{ml}$ of $25 \%$ metaphosphoric acid (MPA), (ref. No.: 253433-4, Sigma-Aldrich, Germany), then $1.4 \mathrm{~mL}$ of distilled water was added, mixed, incubated for 1 hour and centrifuged for 10 min at 3,000 r.p.m then the clean supernatant was removed and used for determination of GSH concentration.

\subsubsection{Liver tissue preparation for molecular analysis:}

Liver tissue were immediately excised and frozen in liquid nitrogen and then in $80^{\circ} \mathrm{C}$ until used for determination of DNA damage using comet assay and intrerleukin1(IL-1) gene expression analysis by qPCR.

\subsection{Analysis:}

\subsubsection{Biochemical analysis:}

Liver tissue L-MDA, superoxide dismutase (SOD), catalase (CAT) and reduced glutathione (GSH) were determined according to the method adapted by Ohkawa et al., (1979), Nishikimi et al., (1972), Aebi, (1984) and Beutler et al., (1963), respectively.

\subsubsection{Molecular analysis:}

Total RNA was isolated from liver tissue of rats using RNeasy Mini Kit (Thermo Scientific, Fermentas, \#K0731) according to the manufacturer's protocol. Following determination of RNA concentration and purity by Quawell nanodrop Q5000 (USA), 5 $\mathrm{mg}$ of total RNA from each sample was reverse transcribed using Quantiscript reverse transcriptase. The produced cDNA was used as a template to determine the relative 
expression of Interleukin-1 gene using StepOnePlus real time PCR system (Applied Biosystem, USA) and gene specific primers. The reference gene, $\beta$ actin, was used to calculate fold change in target genes expression. The thermal cycling conditions, melting curves temperatures, and calculation of relative expression was done. For the treated groups, assessment of $2^{-\Delta \Delta \mathrm{Ct}}$ determined the fold change in gene expression relative to the control.

Forward and reverse primers sequence for real time PCR.

\begin{tabular}{ccc}
\hline Gene & $\begin{array}{c}\text { Forward primer } \\
\left({ }^{\prime} 5----^{\prime} 3\right)\end{array}$ & $\begin{array}{c}\text { Reverse primer } \\
\left(5-\text {--- }^{/ 3}\right)\end{array}$ \\
IL1 & CACCTCTCAAGCAGAGCACAG & GGGTTCCATGGTGAAGTCAAC \\
$\beta$-actin & AAGTCCCTCACCCTCCCAAAAG & AAGCAATGCTGTCACCTTCCC \\
\hline
\end{tabular}

Also, DNA damage was estimated by alkaline single-cell gel electrophoresis (comet assay) according to the protocol described by Singh et al., (1988).

\subsection{Statistical analysis:}

The results were expressed as mean \pm SE using SAS computerized program v. 9.2 (SAS, 2008) program to calculate the

\section{RESULTS}

\subsection{Molecular and Antioxidants parameters:}

The qPCR results (Table 1) revealed a significant up-regulation of IL-1b gene expression level in livers of mycotoxin treated rats as compared to control group. This expression was significantly downregulated following treatment by spirulina.

The obtained results presented in table (2) revealed that, the main value of liver reduced glutathione (GSH) concentration was significantly decreased in mycotoxin group in comparison with control group. Pretreatment with spirulina group (3) significantly increased liver GSH concentration in comparison with mycotoxin group.

The main value of liver (SOD) and (CAT) activities were significantly increased in mycotoxin group in comparison with control group. Pretreatment with spirulina analysis of variance. The data were analyzed using one-way ANOVA to determine the statistical significance of differences among groups. Duncan's test was used for making a multiple comparisons among the groups for testing the inter-grouping homogeneity. Values were considered statistically significant when $\mathrm{p}<0.05$.

group (3) significantly decreased liver SOD and CAT activities when compared with mycotoxin treated rats only.

Exposure of mycotoxin via food in normal rats caused significant increase in the value of liver L-MDA concentration in comparison with control group. Pretreatment with spirulina caused significant decrease in liver L-MDA concentration.

\subsection{DNA damage:}

The obtained results of DNA damage presented in table(3) and the figure (1) showed that, exposure of mycotoxin via food induced significant increase in DNA damage that was indicated by an increase in tail length and tail DNA \% in liver tissue of mycotoxin intoxicated rats as compared to control group. DNA damage was significantly lowered in spirulina treated rats as compared to mycotoxin group. 
Table (1): Changes in relative expression of IL-1b gene in liver tissues of mycotoxin -intoxicated rats after spirulina treatment.
Animal groups
Group I:
Normal control
$1.00^{\mathrm{d}} \pm 0.11$
Group II:
Mycotoxin group $\quad 11.63^{\mathrm{a}} \pm 0.65$
Group III:
Mycotoxin + Spirulina group $\quad 4.11^{\mathrm{c}} \pm 0.27$

IL-1b (Fold change mean \pm SEM)

Mean values with different superscript letters in the same column are significantly different at $(\mathrm{P} \leq 0.05)$. $(\mathrm{n}=3)$ in each group.

Table (2): Effects of spirulina against mycotoxins induced changes on liver tissue GSH, SOD, Catalase and L-MDA in male rats.

\begin{tabular}{lccc}
\hline Parameter & Control group & & \\
\hline GSH(ng/g.tissue) & $4.882 \pm 0.27^{\mathrm{a}}$ & $2.67 \pm 0.20^{\mathrm{c}}$ & $4.174 \pm 0.20^{\mathrm{ab}}$ \\
$\mathrm{SOD}(\mathrm{u} / \mathrm{g} . \mathrm{tissue})$ & $29.04 \pm 2.64^{\mathrm{c}}$ & $57.63 \pm 7.26^{\mathrm{a}}$ & $44.33 \pm 2.41^{\mathrm{b}}$ \\
Catalase(u/g.tissue) & $1.11 \pm 0.04^{\mathrm{b}}$ & $1.37 \pm 0.06^{\mathrm{a}}$ & $0.99 \pm 0.04^{\mathrm{b}}$ \\
MDA(nmol/g.tissue) & $3.36 \pm 0.21^{\mathrm{c}}$ & $6.44 \pm 0.37^{\mathrm{a}}$ & $5.27 \pm 0.4^{\mathrm{b}}$ \\
\hline
\end{tabular}

Data are presented as means \pm Standard Error (S.E)

Means values with different superscript letters in the same row are significantly different at $(\mathrm{P}<0.05)$. $(\mathrm{n}=5)$ in each group.

Table (3): Comet assay parameters obtained by image analysis in rat liver.

\begin{tabular}{cccccc}
\hline Animal groups & $\begin{array}{c}\text { Tailed } \\
\%\end{array}$ & Untailed $\%$ & Tails length $\mu \mathrm{m}$ & $\begin{array}{c}\text { Tail } \\
\text { DNA\% }\end{array}$ & $\begin{array}{c}\text { Tail } \\
\text { moment }\end{array}$ \\
\hline Normal control group & 2 & 98 & $1.29 \pm 0.09^{\mathrm{d}}$ & 1.47 & 1.90 \\
Mycotoxin group & 23 & 77 & $7.68 \pm 0.54^{\mathrm{a}}$ & 7.03 & 54.00 \\
Mycotoxin + Spirulina group & 8 & 92 & $3.32 \pm 0.27^{\mathrm{c}}$ & 2.43 & 8.07 \\
\hline
\end{tabular}

Means within the same column of tail length carrying different superscript letters are significantly different $(\mathrm{P} \leq 0.05)$. 


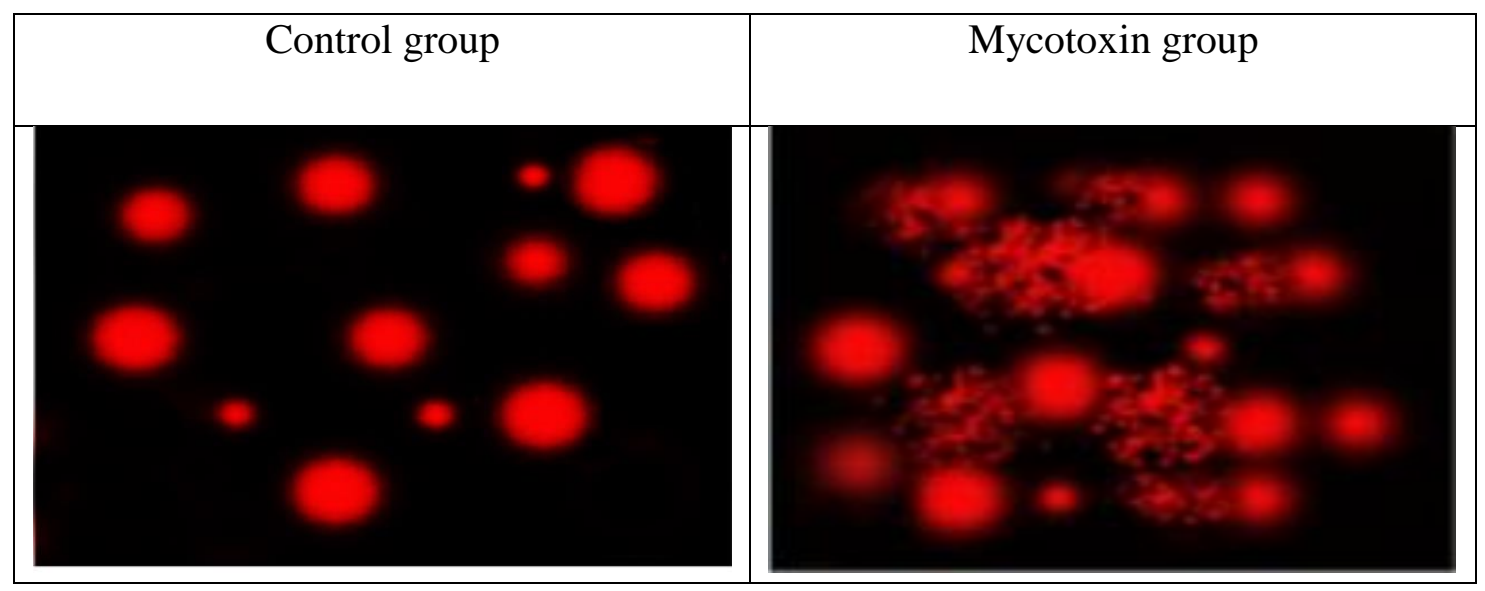

Mycotoxin + Spirulina group

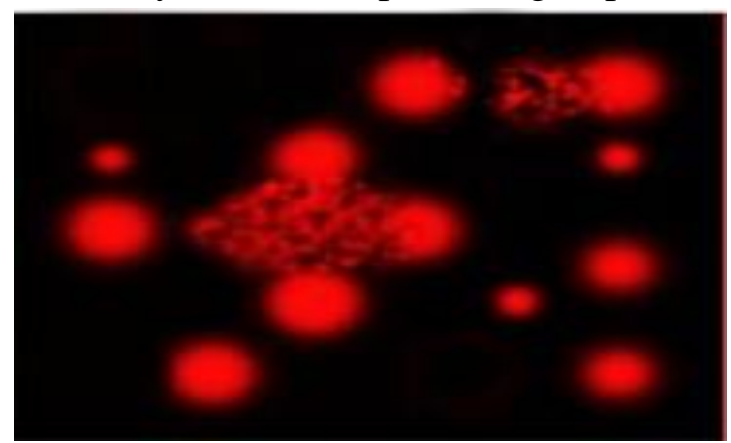

Fig.(1): Photomicrographs representation of DNA damage, using comet assay, in normal control group, mycotoxin group and Spirulina treated group.

\section{DISCUSSION}

Mycotoxins are toxic chemicals formed as secondary metabolites of fungi. They are frequently consumed in food products from crops origin, such as cereals, nuts, dried fruit, spices, oil seeds, beans, fruit, beer and wine (Turner et al., 2009). The contamination of animal, fish and human feeds with mycotoxins represent a worldwide problem constituting a real threat to the health of livestock for animals, aquaculture industry and human by the continuing intermittent occurrence in their feeds (Vekiru et al., 2007). Spirulina can be a very interesting natural source of new compounds with biological activity that could be used as functional ingredients. The cyanobacterium Spirulina is used as food by humans because of its chemical composition, which has high quality and quantity of proteins, essential amino acids, minerals, polyunsaturated fatty acids and vitamins. It has been reported to prevent oxidative damage, and hence can indirectly reduce cancer formation in human body (Arijit et al., 2012).

The obtained qPCR results revealed a significant up-regulation of IL-1b gene expression level in livers of mycotoxin rats as compared to control group. These results are nearly similar with the recorded data of Albarenque et al., (2001) who suggested that, the expression of pro-inflammatory cytokines IL-1b was increased in the T-2 toxin-treated cells cultures throughout the experimental period. Likewise, Abdel-Wahhab et al., (2006) postulated that, the ingestion of aflatoxins B1 (AFB1) significantly increased IL-1b suggesting that AFB1 preferentially affects macrophage functions. The expression of IL-1b gene was significantly down- 
regulated following treatment by spirulina. This suggestion was supported the findings of Hörl, (2010) who recorded that, Spirulina extract also extensively inhibit the inflammatory cascade by effectual modulation of inflammatory cytokines (IL-1), thus curbing the further exacerbation of mycotoxin renal injury mediated by inflammatory cytokines.

The obtained results revealed that, the main value of liver reduced glutathione (GSH) concentration was significantly decreased in mycotoxin group in comparison with control group. These results were in a harmony with those of Stockmann-Juvala et al., (2004) who concluded that, oxidative stress and apoptosis may be involved in the neurotoxicity induced by AFB1, which caused disturbance of GSH generation. According to Umarani et al., (2008) a significant reduction in the levels of hepatic GSH was observed in the AFB1 group. This depletion of liver GSH may be attributed to its conjugation with the electrophilic metabolites. Pretreatment with spirulina group significantly increased serum GSH concentration in comparison with mycotoxin group. These results were nearly similar with Hassan et al., (2012) who evaluated the ability of Spirulina to counteract oxidative stress in rats with mycotoxin-induced aflatoxicosis. Supplementation with Spirulina led to a significant reduction in aflatoxin-induced oxidative stress, as revealed by increased GSH content. Meanwhile, Abd El-Baky et al., (2009) recorded that, Spirulina platensis maintained the activity of cellular antioxidant enzymes under oxidative stress and increased the level of reduced glutathione in these cells.

The results of the current study revealed that, mycotoxin treated rats significantly increased liver superoxide dismutase (SOD) and catalase (CAT) activities in comparison with control group. These results came in accordance with the recorded data of Chaudhary and Rao (2010) who reported that, mycotoxin treated animals increasing ROS generation showed significant increase in the gene expression profile of antioxidant enzymes SOD and CAT. Pretreatment with spirulina significantly decreased liver SOD and CAT activity when compared with mycotoxin treated rats only. Likely, Qing et al., (2003) found that, the activity of two antioxidant enzymes contained within Spirulina maxima, superoxide dismutase (SOD) and catalase (CAT), increased under conditions of light stress, although SOD was more sensitive to external stress than CAT.

The recorded data showed significant increase in the value of liver tissue L-MDA in mycotoxicated rats when compared with normal control group. These results were agree well with those of Chaudhary and Rao, (2010) who reported that, T2 toxin-treated rats showed a timedependent increase in ROS generation and marked increase in lipid peroxidation in the kidney during oral administration of Aflatoxin for 30 days.

Pretreatment with spirulina caused significant decrease in liver L-MDA concentration. These results were similar with the recorded data of Yoshikawa and Naito, (2002) who stated that, treatment with Spirulina significantly reduced oxidative stress and L-malondialdehyde in mycotoxin intoxicated rats. Reactive oxygen species (ROS) attacked and damaged molecules in biological systems, leading to oxidative stress and various disorders and diseases occur. The antioxidant potential of Spirulina species and protective effects were mediated by phycocyanins, $\beta$-carotene, and other vitamins and minerals contained within Spirulina (Abdel-Daim et al., 2013). Moreover, Bermejo et al., (2008) reported that, phycocyanin appear to inhibit the generation 
of hydroxyl and peroxyl radicals, as well as lipid peroxidation.

\section{CONCLUSION}

From the obtained results it could be concluded that, Spirulina Platensis protect the liver from mycotoxin induced oxidative stress and tissue damage. The protective effect of spirulina might be mediated by adjustment of inflammatory mediators and increasing antioxidants as well as attenuating oxidant/antioxidant imbalance. It seems likely that spirulina enriched in phenolic compounds contains no hepatotoxin. So, its possible potential as a promising natural therapeutic agent in liver damage and other diseases associated with oxidative stress.

\section{REFERENCES}

Abd El-Baky,H.H., El Baz,F.K. and ElBaroty, G.S. 2009. Enhancement of antioxidant production in Spirulina platensis under oxidative stress.

Abdel-Daim,M.M., Abuzead,S.M. and Halawa, S.M. 2013. Protective role of Spirulina platensis against acute deltamethrin-induced toxicity in rats. PLoS ONE 8(9):e72991

Abdel-Wahhab,M.A. ， Ahmed,H.H. and Hagazi,M.M.2006. Prevention of aflatoxin B1- initiated hepatotoxicity in rat by marine algae extracts. J.Appl.Toxicol.26, 229-238.

Acta Physiol Plant 31:623-631

Aebi, H.1984. Catalase in vitro, Methods Enzymol 105, 121-126.

Albarenque,S. M., Suzuki, K., Nakayama, H. and Doi, K. 2001. Kinetics of cytokines mRNAs expression in the dorsal skin of hypotrichotic WBN/ILA-Ht rats following topical application of $\mathrm{T}-2$ toxin, Exp. Toxicol.Pathol. 53: 271274.

Arijit, D., Angayarkanni, J., Sourav Bhattacharya, Palaniswamy, M. 2012. Evaluation of process parameters influencing aflatoxin B1 synthesis from Aspergillus flavus MTCC 2798 using rice straw under submerged fermentation. Int. J. Pharmacy Biol. Sci., 2: 94-105.

Ayçiçek, H., Aksoy,A. and Sayg1,S. 2005. Determination of Aflatoxin Levels in Some Dairy and Food Products which Consumed in Ankara, Turkey. Food Control, Vol. 16, p. 263-266.

Bermejo, P., Piُero, E.and Villar,M. 2008. Iron-chelating ability and antioxidant properties of phycocyanin isolated from a protean extract of Spirulinaplatensis. Food Chem 110(2):436-445

Beutler, E., Duron, O. and Kelly, M.B. 1963. Improved method for the determination of blood glutathione, J. Lab Clin. Med. 61:882-88

Chaudhary, M. and Rao, P.V.2010. Brain oxidative stress after dermal and subcutaneous exposure of T-2 toxin in mice. Food Chem. Toxicol., 48, 34363442 .

Cohen, Z. 1997. The chemicals of Spirulina, In: Vonshak, A. (Ed), Spirulina platensis (Arthrospira): Physiology, Cell-biology and Biotechnology, Taylor and francis Ltd, 175-204.

Colla, L.M., Muccillo-Baisch, A.L., Costa, J.A.V. 2008. Spirulina platensis Effects on the Levels of Total Cholesterol, HDL and Triacylglycerols in Rabbits Fed with a Hypercholesterolemic Diet. Brazilian Arch Biol and Technol. 2008; 51:405-411.

Fink-Gremmels, J. 1999. Mycotoxins: Their implications for human and animal health. Vet.Q. 21:115-120.

Hassan, A.M., Abdel-Aziem, S.H. and AbdelWahhab, M.A. 2012. Modulation of DNA damage and alteration of gene expression during aflatoxicosis via dietary supplementation of Spirulina (Arthrospira) and Whey protein 
concentrate. Ecotoxicol Environ Saf 79:294-300

Hörl, W.H. 2010. Nonsteroidal antiinflammatory drugs and the kidney .Pharmaceuticals $3: 2291-2321$

Hussein, H.S. and Brasel, J.M. 2001. Toxicity, metabolism, and impact of mycotoxins on humans and animals. Toxicology. 167:101-134.

Karkos, P.D., Leong, S.C., Karkos, C.D., Sivaji, N. and Assimakopoulos, D.A. 2008. Spirulina in Clinical Practice Evidence-Based Human Applications. Evid Based Complement Alternat Med.:1-4. eCAM.

Komárek, J. and Hauer, T. 2009. Worldwide electronic publication. Univ. of South Bohemia and Inst of Botany AS CR. CyanoDB.cz - On-line database of cyanobacterial genera.

Nishikimi, M., Roa, N.A. and Yogi, K. 1972. Measurement of superoxide dismutase Biochem. Bioph. Res. Common., 46, $849-854$.

Ohkawa, H., Ohishi, W., and Yagi, K. 1979. Assay for lipid peroxides in animal tissues by thiobarbituric acid reaction. Anal Biochem 95(2):351-8.

Qing, R., Ye, H., Lan, L. and Fu, H. 2003. Study of the activity of two antioxidant enzymes of Spirulina maxima under excessive light stress. J Sichuan Univ 40(3):565-569.

SAS . 2008. Statistical analysis system :

User's guide v. 9.2, Inst. Inc., Cary

N.C., USA.

Singh, N., McCoy, M., Tice, R., Schneider, E. 1988. A simple technique for the quantitation of low levels of DNA damage in individual cells. Exp Cell Res 175: 184-191.

Stockmann-Juvalla, H., Mikkola, J., Naarala, J., Loikkanen, J., Elovaara, E. and Savolainen, K.2004. Oxidative stress induced by fumonisin B1 in continuous human and rodent neural cell cultures. Free Radic. Res., 38, 933-942.

Turner, N. W.,Subrahmanyam, S., and Piletsky, S. A. 2009.Analytical methods for determination of mycotoxins: A review. Analytica Chimica Acta, 632(2), 168-180.

Umarani, M., Shanthi, P. and Sachdanandam, P. 2008. Protective effect of Kalpaamruthaa in combating the oxidative stress posed by aflatoxin B(1)-induced hepatocellular carcinoma with special reference to flavonoid structure-activity relationship. Liver Int;28:200-13.

Vekiru, E., Fruhauf, S., Sahin, M., Ottner, F., Schatzmayr, G. and Krska, R. 2007. Investigation of various adsorbents for the irability to bind aflatoxin B1 .J. Mycotoxin research ,Vol.23 No.1, PP 27,33.

Yoshikawa, T. and Naito, Y. 2002. What is oxidative stress. JMAJ 45(7):271-276 